\title{
Laser-pulse-vaporization of refractory materials
}

\author{
Donald R. Olander \\ Materials and Chemical Sciences Division, Lawrence Berkeley Laboratory and the Department of \\ Nuclear Engineering, University of California, Berkeley, CA 94720
}

\begin{abstract}
Recent experimental and theoretical developments concerning the interaction of laser light with refractory solids are reviewed, with emphasis on long, low-power density pulses. Compared to short, high-power density irradiation, long pulses produce principally thermally-driven evaporation which can be related to the thermochemical properties of the heated surface. Surface temperature measurement and calculation methods are outlined and the use of mass spectrometry to analyze the vapor is reviewed.
\end{abstract}

\section{INTRODUCTION}

The incidence of short nanosecond- to millisecond-wide pulses of laser light on solids serves two functions. First, it is used to produce desirable changes in the substrate, including welding, annealing and alloying[1,2]. Second, laser irradiation can remove solid, either by a thermally-activated evaporation process or by an explosive ejection of material sometimes called ablation. The second application is the subject of this review.

In addition to the well-established laser machining operations(cutting, welding, drilling), two examples of practical application of laser vaporization are production of clusters of many materials and production of thin films of high- $T_{c}$ superconducting ceramics. In the former application, the laser-generated vapor is condensed in an inert carrier gas and transported to detection instrumentation[3]. In the latter application, targets of bulk material are vaporized in vacuum and deposited as superconducting films on suitable substrates[4]. In this application, congruency of vaporization, meaning that the composition of the vapor is equal to that of the solid, is critical. Finally, laser vaporization(or ablation) is the basis of a commercial ion source for mass spectrometric analysis of solids .

Laser heating can also be used as a convenient surface heat source for fundamental studies that simulate vaporization process occuring in systems that are normally heated by other means. For example, the very high temperatures that can occur in a nuclear reactor core during a severe accident are produced by a combination of nuclear heating from decay of fission products and chemical heating from steam-oxidation of the metallic components of the fuel element. Temperatures as high as $3000^{\circ} \mathrm{C}$ can be attained, and vaporization of of the $\mathrm{UO}_{2}$ fuel and refractory fission products is a major ingredient in analysis of such an accident. In another example, disruptions of the thermonuclear plasma in tokamak-type fusion reactors results in rapid energy deposition on plasma-facing components, which are constructed of or lined with refractories such as graphite, silicon carbide or tungsten. In this case heating is caused by a combination of high-energy ions and electrons and $\mathrm{X}$ rays.

Vaporization of the wall materials can adversely affect the power balance in the plasma and must be controlled. In a final example, aerodynamic frictional heating during re-entry of space vehicles is accomodated by heat shields fabricated of graphite composites, which are designed to vaporize in a controlled fashion at the peak temperatures achieved.

In these three cases of diverse modes of heating of a solid, substitution of pulsed-laser heating provides a useful method for studying the kinetic and thermodynamic aspects of the vaporization process that cannot be achieved using the actual heat source. The laser can be focussed on to a spot of small diameter(typically several millimeters) on the surface, which as a result is driven briefly to very high but controllable temperatures $(4000-5000 \mathrm{~K})$. The laser provides a localized, mainly surface heat source, so that the target serves as its own crucible. Conventional steady-state means for investigating vaporization processes, such as Knudsen-cell or transpiration methods, require heating of bulk structures to high temperature. These methods are limited to maximum temperatures of about $3000^{\circ} \mathrm{C}$ by the lack of container materials to hold specimens at higher temperatures. Usually, chemical reaction between specimen and container dictate a lower useable maximum temperature than that determined solely be container melting. Both of these restrictions are absent in laser heating.

Except where an ambient gas atmosphere is required for a particular application, most laser evaporation 
experiments are conducted in vacuum. This environment aids in maintaining surface cleanliness, allows use of in situ detectors such as mass spectrometers and surface analyzers, and avoids vapor interactions with another gaseous species.

Measurement of vaporization by laser heating is not without severe drawbacks. Principal of these is the difficulty in measuring the temperature of the surface during the heating transient. This is possible only for relatively long pulses(order of milliseconds) characteristic of conventional-mode Nd-glass lasers, but conventional optical pyrometry is not feasible for nanosecond pulses from an excimer laser. A second difficulty is the variety of physical processes which can occur during the interaction of the laser with the solid. The surface of the target usually melts and because of the recoil pressure of the vaporizing material, radial flow of the liquid can occur. The molecules in the vapor may collide with one another, changing the blowoff from a free-molecule flow to a gasdynamic expansion. At sufficiently high vapor densities, the laser may interact with the plume thereby creating a plasma and shielding the surface from the incoming laser light. The multitude of processes which can occur and their dependence on laser power, pulse length, wave length and material properties such as thermal conductivity and vapor pressure, renders clean-cut physical interpretations of the results problematic.

Despite these difficulties, pulse-laser heating of solids has become an important tool in studying the vaporization process at temperatures unattainable by conventional means.

\section{REGIMES OF LASER-SOLID INTERACTIONS}

The absorbed power density is a convenient although not unique measure for categorizing the nature of the laser-solid interaction. This quantity, together with the pulse duration and the reflectivity of the surface, determines the absorbed energy density. The consequences of energy absorption depend on a wide variety of physical and chemical properties of the target material, chief among which are the thermal diffusivity, the vapor pressure, and the optical absorbance. These properties govern the depth of penetration of the thermal front, the maximum surface temperature and the density of the vapor just above the surface.

In this review, only laser power densities which produce significant vaporization will be considered. At power densities from approximately $10^{3}$ to $10^{4} \mathrm{~W} / \mathrm{cm}^{2}$ only modifications of the target, such as melting or annealing, occur.

\section{Low laser power density - long pulse time}

Power densities less than about $10^{5} \mathrm{~W} / \mathrm{cm}^{2}$ lasting for approximately a millisecond are sufficient to heat the surface of poorly-conducting materials such as oxides to temperatures in the vicinity of $4000 \mathrm{~K}$. To reach comparable temperatures on a metal surface, longer pulses or higher power densities are required to compensate for the greater heat loss by conduction into the bulk solid.

Under these conditions, the main mechanism of material removal is ordinary vaporization into a vacuum. The kinetics of this process were described many decades ago by Langmuir by application of the principle of detailed balancing. This principle states that at equilibrium, the rates of all foward and reverse processes must be equal. Thus, the rate of evaporation of a solid or liquid in contact with its equilibrium vapor is equal to the rate of impingement of molecules from the gas on the surface, which is calculable from gas kinetic theory, times the probability that the molecules stick, or condense. Langmuir then assumed that the vaporization rate remains unchanged when the equilibrium vapor is replaced by a vacuum. The resulting Hertz-Langmuir formula,

$F=\frac{\alpha P\left(T_{S}\right)}{\sqrt{2 \pi m k T_{s}}}$

is the basis for measurement of the vapor pressure-temperature relationship for refractory metals such as tungsten. In $\mathrm{Eq}(1), \mathrm{F}$ is the vaporization flux from the surface, $\mathrm{T}_{\mathrm{s}}$ is the surface temperature, which is in general time-dependent, $\mathrm{m}$ is the mass of the evaporating molecule and $\mathrm{k}$ is the Boltzmann constant. The parameter $\alpha$ is the evaporation(or condensation) coefficient, which accounts for imperfect sticking of impinging molecules on the surface. It is usually close to unity unless the vapor species has an appreciably different molecular structure from that in the condensed phase. Crystalline arsenic, for example, has a rhombohedral crystal lattice structure but the vapor consists of tetrahedral $\mathrm{As}_{4}$ molecules. The sluggish rate of atomic rearrangement prior to vaporization is 
manifest as a very low evaporation coefficient for this element[5].

If the solid contains more than one element, say $A$ and $B$, and evaporates as molecules $A_{i} B_{j}$, the flux of a particular species characterized by the stoichiometric numbers $\mathrm{i}$ and $\mathrm{j}$ is given by:

$$
F_{i j}=\frac{\alpha_{i j} P_{i j}\left(T_{s}, x_{s}\right)}{\sqrt{2 \pi m_{i j} k T_{S}}}
$$

where $P_{i j}$ is the partial pressure of $A_{i} B_{j}(g)$ in equilibrium with the condensed phase at the surface of temperature $T_{s}$ and composition $\mathrm{x}_{\mathrm{s}}$ (expressed as the atom fraction of $\mathrm{A}$, for example). The dependence of the vaporization rate of each molecular species on the surface A-to-B ratio means that all vaporization fluxes depend on the rate at which A or B can be supplied to the surface by diffusion from the bulk in order to replenish the loss by vaporization[6].

The less-volatile constituent of the condensed phase becomes enriched at the surface with respect to the bulk composition. This is associated with incongruent vaporization, in which the composition of the vapor is different from that of the bulk condensed phase. For example, the vapor flux from uranium carbide is rich in $\mathrm{U}(\mathrm{g})$, with smaller quantities of $\mathrm{UC}_{2}(\mathrm{~g})$ and $\mathrm{C}_{3}(\mathrm{~g})$; vacuum vaporization of the superconducting oxide $\mathrm{YBa}_{2} \mathrm{Cu}_{3} \mathrm{O}_{7}$ first produces essentially pure molecular oxygen, followed by the oxides and atoms of copper, then the vapor species of the refractory oxides of barium and yttrium.

Incongruency occurs only in the initial stages of vacuum vaporization. If the surface is maintained at a constant temperature (greater than that of the bulk), the total vaporization rate, and hence the surface recession speed, also approach steady-state values. When this state has been achieved, a simple mass balance over the moving surface requires that the compositions of the solid and the vapor be equal. This condition of "forced congruent

vaporization" [7] is achieved at times greater than the characteristic time $\left(D / u^{2}\right)^{1 / 2}$, where D is the mutual diffusion coefficient in the binary A-B condensed phase(which is usually liquid for significant vaporization to occur) and $u$ is the speed of surface recession. The latter is related to the vaporization fluxes by[6]:

$$
u=\left(M_{A} \Sigma i F_{i j}+M_{B} \Sigma j F_{i j}\right) / \rho
$$

where $\rho$ is the mass density of the condensed phase at the surface, assumed to be independent of the $A / B$ ratio, and $M_{A}$ and $M_{B}$ are the atomic weights of elements $A$ and $B$, respectively. Forced congruent vaporization can also be achieved in periodic pulsed evaporation.

Thus, equilibrium vacuum vaporization of multicomponent solids by laser or other heat sources involves a complex coupling of:

- heat and mass transport in the near-surface regions of the condensed phase, which governs the surface temperature and surface composition

- vaporization kinetics according to the Hertz-Langmuir theory

- the thermochemistry of the material, by which the equilibrium pressures in Eq(2) are determined.

\section{Effect of molecular collisions in the gas phase}

If the laser power density is such that only modest surface temperatures are reached and the vapor pressure of the material is low at these temperatures, the molecules emitted from the target do not undergo collisions in the vapor phase. This is the free-molecule flow regime. Far from the surface, a density-sensitive detector should observe a cosine angular distribution, a speed distribution corresponding to a Maxwell distribution characteristic of the surface temperature, and a total evaporation rate given by $\mathrm{Eq}(1)$.

At input power densities roughly between $10^{5}$ and $10^{6} \mathrm{~W} / \mathrm{cm}^{2}$, collisional effects in the vapor become significant. The transition between the free-molecule and the gasdynamic vapor blowoff regimes can be studied by Monte Carlo methods[8] or by the simpler test-particle method[9]. In the latter method, the number-density distribution of vapor molecules above the evaporating surface is first calculated assuming collisionless flow. For a finite source, this distribution varies from the density corresponding to one half of the equilibrium vapor pressure at the surface temperature to zero far downstream from the surface. Then a test particle is emitted from the center of the evaporating surface along the normal. Knowing the atomic scattering cross section of the vapor molecules, the probability that the test particle escapes from the cloud without undergoing a collision is determined as a function of surface temperature(or equivalently of the vapor pressure). Figure 1 shows the results of such a calculation for steady-state evaporation of uranium dioxide from an 8- $\mathrm{mm}$-diameter heated spot.The collision cross section was 
taken to be $0.43 \mathrm{~nm}^{2}$. The curves correspond to various levels of rigor in the analysis; curves $\mathrm{c}$ and $\mathrm{d}$ probably bracket the exact result. At low temperatures, the escape probability is unity, which signifies collisionless flow. At high temperature, the probability of escape is zero, meaning that the flow is of the gasdynamic type similar to that of a gas jet expanding from a high pressure reservoir through an orifice into a vacuum. Over a $400 \mathrm{~K}$ range, the escape probability drops from 1 to 0 . The transition from free-molecule to gasdynamic flow can be defined to occur when the escape probability is one half, which, for $\mathrm{UO}_{2}$, is about $2600 \mathrm{~K}$.

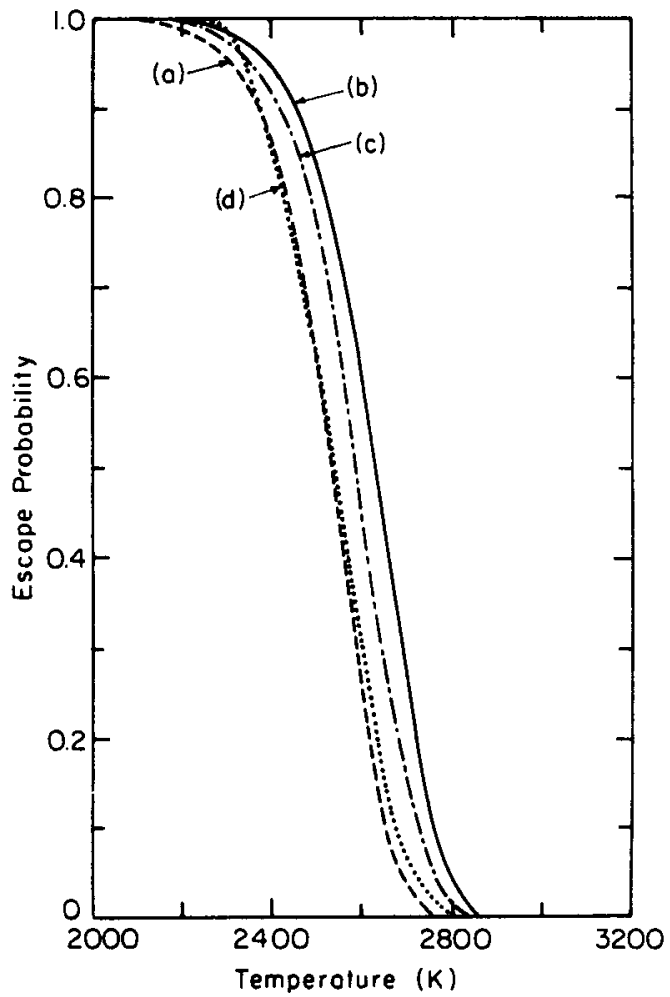

Fig. 1. Escape probability as a function of surface temperature for steady vaporization of uranium dioxide from an $8 \mathrm{~mm}$ diameter heated surface into vacuum. The curves labeled a-d represent various approximations in the calculation (after Ref. 9)

The calculation shown in Fig. 1 is for steady evaporation. A similar analysis can be performed for the temperature transient produced by a heat pulse which has a triangular temporal shape. For a pulse width of $0.2 \mathrm{~ms}$, the $50 \%$ escape probability occurs at a temperature about $50 \mathrm{~K}$ higher than that for the steady-state case.

The analysis leads to the following rule of thumb for approximating the $50 \%$ escape probability temperature for other conditions or other materials: the product of the equilibrium vapor number density at the surface temperature, the collision cross section, and the radius of the heated spot is approximately 2.5[9].

The effect of intermolecular collisions in the vapor in the gasdynamic regime is to transform random thermal energy of the vapor molecules into translational kinetic energy along the normal to the surface. This phenomenon occurs in both expansion of a high-pressure gas from an orifice[10] or of the blowoff from a laser-heated spot on a solid or liquid surface[11,12]. However, the analogy between these two situations is not exact because in the latter a thin transition zone known as the Knudsen layer separates the surface and the beginning of the adiabatic expansion(Fig. 2). In this layer, the translational distribution along the normal ( $z$ direction) changes from a half-Maxwellian

$$
f_{F M} \propto \exp \left(-\frac{m v_{z}^{2}}{2 k T_{s}}\right) \quad\left(v_{s} \geq 0\right)
$$

at the surface to the full Maxwellian

$$
f_{G D} \propto \exp \left(-\frac{m\left(v_{z}-v_{K}\right)^{2}}{2 k T_{K}}\right) \quad\left(-\infty<v_{z}<\infty\right)
$$


on the downstream side of the Knudsen layer. In $\mathrm{Eq}(5), \mathrm{v}_{\mathbf{z}}$ is the molecular speed component in the $\mathrm{z}$ direction, $\mathrm{v}_{\mathrm{K}}$ is the flow speed about which the distribution is centered and $T_{K}$ is the temperature characterizing width of the velocity distribution along the normal to the surface.

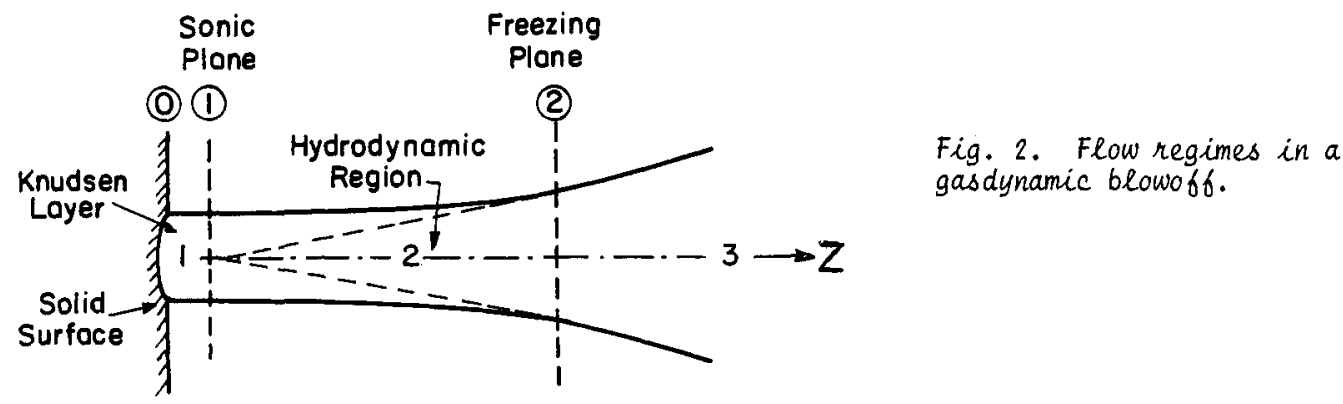

The adiabatic expansion following the Knudsen layer narrows the distribution somewhat but does not appreciably affect the mean flow speed[13]. The number density of the vapor decreases roughly as the inverse square of the distance. The flow becomes supersonic in the sense that the flow velocity exceeds the local sound velocity. In addition, the flow velocity is greater than the mean speed of the Maxwellian distribution at the surface temperature by a factor ranging from 1.26 to 1.64[13]. The gasdynamic flow is "cold" because $T_{K}<T_{s}$ as a result of transformation of thermal motion to directed flow. The angular distribution of the flow is more sharply peaked about the normal than that in a collisionless vapor; the distribution varies as $\cos ^{n} \theta$ where $\theta$ is the angle from the surface normal and $n>2$.

At the freezing plane in Fig. 2, the gas density becomes so low that intermolecular collisions cease, and the gas continues to expand in free-molecule flow.

With respect to a detector downstream of the surface, the principal effect of the modifications of the flow due to the combined effects of the Knudsen layer and the gasdynamic expansion is to speed up the gas by 26 to $64 \%$. The gas density sensed by the detector, however, is not greatly affected. In both free-molecule and gasdynamic expansions, molecules leave the surface at a rate given by $\mathrm{Eq}(1)$. In the latter case, three effects must be considered. First, the recondensation on the surface which occurs by backscattering of molecules emitted from the surface on the outer edge of the Knudsen layer reduces the vaporization flux by $18-25 \%$ Second, for a fixed evaporation rate, the higher flow speed proportionately reduces the number density to which the detector responds. Finally, the sharper-than-cosine angular distribution provides more molecules along the surface normal where the detector is usually located. The combination of three effects leads to very little change in the measured number density from the gasdynamic expansion compared to what would be observed for a collisionless flow[14]. Thus, the principal observable difference between a gasdynamic expansion and a free-molecule flow vaporization process is the higher mean speed of the former. This conclusion ignores the possibility that the gasdynamic expansion may result in a highly supersaturated vapor in which nucleation and growth of clusters may occur.

\section{High laser power density - short pulse times}

Although the principal concern here is the nature of the laser-solid interaction during low power density pulses of millisecond lengths, a brief review of the type of interaction when the power density exceeds $10^{7}$ to $10^{8} \mathrm{~W} / \mathrm{cm}^{2}$ is in order.

At low power densities, the interaction has an essentially thermal character, in the sense that the material removal rate follows the vapor pressure according to $\mathrm{Eq}(1)$. For higher power densities delivered in pulse times on the order of tens of nanoseconds(typical of excimer lasers) a variety of other, nonequilibrium, mechanisms assume greater importance than thermally-driven emission. These are generally of the "explosive" type involving emission of large masses of material rather than individual molecules.

One of the earliest of these mechanisms to be proposed is the recoil pressure model proposed by Ready[15] and others[16]. In this process, the rapid, initially thermally-driven vaporization flux creates a back pressure on the surface in much the same way that the flow from a hose produces a force on the structure(or person) holding the 
hose. The back pressure created by the departing vapor is about one half of the vapor pressure at the surface temperature[17]. This recoil pressure can contain the hot liquid surface and prevent further evaporation, thereby producing a superheated liquid. As the pulse ends, the restraining vapor escapes and rapid nucleation of vapor bubbles in the superheated liquid leads to explosive release in the form of ejected droplets

A modification of this model involves subsurface heating of the liquid[18,19]. If the condensed phase does not strongly absorb the incident laser light, in-depth heating rather than purely surface heating occurs. The energy deposited decreases exponentially with depth in the condensed phase with a characteristic length equal to the reciprocal of the optical absorption coefficient. The combination of in-depth energy deposition and cooling of the surface due to the necessity of supplying the heat of vaporization to sustain the initially thermal evaporation results in a maximum temperature below rather than at the surface. The high vapor pressure of the material at the location of the temperature maximum drives nucleation and growth of vapor bubbles, thereby leading to an explosive release of both the gas and entrained liquid.

Droplet emission has been clearly demonstrated by scanning electron micrographs of the surface of laser irradiated gold[20,22]. The model proposed involves heating and thermal expansion of small protuberances or asperities on the surface which, because of thermal expansion, tend to be driven away from the surface. If the kinetic energy of the minute liquid hills exceeds the enegy required to create additional surface area to form a detached drop, the latter are ejected from the heated surface.

Emission of chunks of solid can occur in metals which do not even reach the melting point during the laser pulse[20,21,23]. In this process, the steep temperature gradients in the solid generated thermal stresses that exceed the fracture stress. The result is creation of flakes of solid which become detached from the surface. Tungsten and alumina exhibit this type of behavior[20].

In addition to the athermal material removal processes, absorption of the incident laser light by the vapor can also occur at high laser power densities. The hot, high-density vapor plume produced by thermal vaporization can become ionized by a variety of mechanisms and ultimately create a full-fledged plasma that strongly absorbs incident laser light. Free electrons and ions are produced in any hot gas by the Saha equilibrium

$$
A(g)=A^{+}(g)+e
$$

which has a Boltzmann-factor temperature dependence involving the ionization energy of the neutral atom or molecule A. In addition, electrons and ions can be emitted directly from the condensed phase at a rate governed by Richardson's equation:

$$
\mathrm{J}=B T^{2} \mathrm{e}^{-\mathrm{W} / \mathrm{T} T}
$$

where $\mathrm{W}$ is the electron or ion work function of the solid or liquid[24].

Mechanisms by which laser light is absorbed by the vapor include an inverse Bremstrahlung process, whereby free electrons in the vapor interact directly with the laser photons[25]; bound-free adsorption, in which bound electrons are pumped to the inoization level by absorption of one or more photons[2]; and bound-bound transitions driven by laser photon absorption in the atoms or molecules of the vapor[26]. Ignition of plasmas in atmospheric-pressure air occurs at power densities as low as $10^{6} \mathrm{~W} / \mathrm{cm}^{2}$ [27], so comparable thresholds would be expected in vapor plumes which attain similar number densities. For most systems, the laser power density at which vapor absorption of laser light begins is probably closer to $10^{8} \mathrm{~W} / \mathrm{cm}^{2}[25]$. Vapor-absorption processes can so efficiently shield the surface from the laser light that the material removal rate per laser pulse actually decreases as the power density increases[25]. However, target heating even with strong vapor absorption can continue by thermal conduction or radiation from the plasma to the surface. In metals, ultraviolet reradiation from the plasma can even enhance energy absorption by the surface if the laser is in the infrared where absorptance is low[27].

\section{EXPERIMENTAL ASPECTS - LOW POWER DENSITIES}

A large number of diagnostic tools have been employed for characterizing the nature and extent of laser-evaporated plumes. These include:

- measurement of the depth of the depression in the target material after a number of identical laser shots. This 
information, along with measured surface temperature during the transient permits determination of the vapor pressure by use of $\mathrm{Eq}(1)$ [24]. Alternatively, if the vapor pressure-temperature relation is known for the target material, the crater depth serves as a measure of the temperature[20,28]. The amount of material removed is an integral measure of the consequence of the laser-solid interaction.

- Vapor condensation on collection disks surrounding the target. This technique is also an integral measure of the interaction. In conjuction with neutron activation analysis, it is useful for measuring the total quantity vaporized as well as the angular distribution of the plume[29]. The condensate on the disks can also be examined by scanning electron microscopy or replica transmission electron microscopy to determine if visible(micron-size) droplets were emitted from the surface or were formed by condensation in the gasdynamic expansion[29,30].

- In situ mass spectrometry. This technique is useful in assessing whether the ratios of molecular species emitted during the pulse conforms to thermochemical expectations[14,24,29,31,32].

- Recoil momentum measurements by ballistic pendulum. Both the target and a collection disk can be suspended by fine wires and their deflection following a laser pulse on the target measured. This technique can be used to provide an average "temperature" and vapor pressure during the pulse[33].

- Charged particle(ions, electrons) detection[24] or deflection[29]. Faraday probes, deflecting magnets and grids and the mass spectrometer proper can be used to assess the contributions of ions and neutral species in the vapor.

- Laser-induced fluorescence analysis of the vapor plume using a separate pulsed dye laser probe which traverses the plume but does not strike the surface[28]. This technique also requires a spectrophotometer to analyze the light emitted by the excited vapor molecules. The technique is useful for determining the rotational and vibrational temperatures of species in the vapor.

- Time-of-flight techniques using a quadrupole mass spectrometer[29] or spectroscopic methods[28]. Measurement of the time required for the vapor molecules to traverse a known distance in vacuum permits determination of their average speed. It is of particular interest to know whether this speed is that characteristic of a Maxwellian distribution at the surface temperature, which would indicate free-molecule flow, or whether the flow has been accelerated by the gasdynamic expansion and changes occuring as the Knudsen layer is crossed[13,29].

- Use of a time-of-flight mass spectrometer(not to be confused with the time-of-flight technique described in the preceding paragraph). This instrument differs from a quadrupole mass spectrometer because separation of the masses is effected by drift down a long tube. It is especially useful in analyzing very high molecular weight molecules, and is probably the only instrument that can detect clusters due to condensation in gasdynamic vaporization. It has been used for this purpose with supersonic molecular beams for some time[3]

A typical experimental setup using quadrupole mass spectrometric detection is shown in Fig. 3. Its features and the type of measurements it permits are discussed below.

\section{Surface temperature measurement and calculation}

As shown in Fig. 3, an optical pyrometer is aimed at the same spot on the target that is irradiated by the laser beam. Either specially made pyrometers [34] or commercial automatic optical pyrometers [29] can be used for this purpose. Direct temperature measurement by optical pyrometry is applicable only to slow transients(i.e., pulse widths of about $100 \mu \mathrm{s}$ or greater). Accurate temperature measurement requires careful attention to a number of potential problems.

- Appropriate preamplification of the output signal from the electron multiplier of the pyrometer is necessary to avoid signal distortion by cables leading to the recording device, which is usually an analog-to-digital recorder.

- Means of extending the calibration of the pyrometer to temperatures well beyond those available in standard NBS-calibrated sources. Black-body sources are available up to $3000^{\circ} \mathrm{C}$; calibration to higher temperatures can be accomplished using Planck's law.

- The emissivity of the surface(in both the solid and liquid states) must be known in order to convert the brightness temperature to the true temperature. 


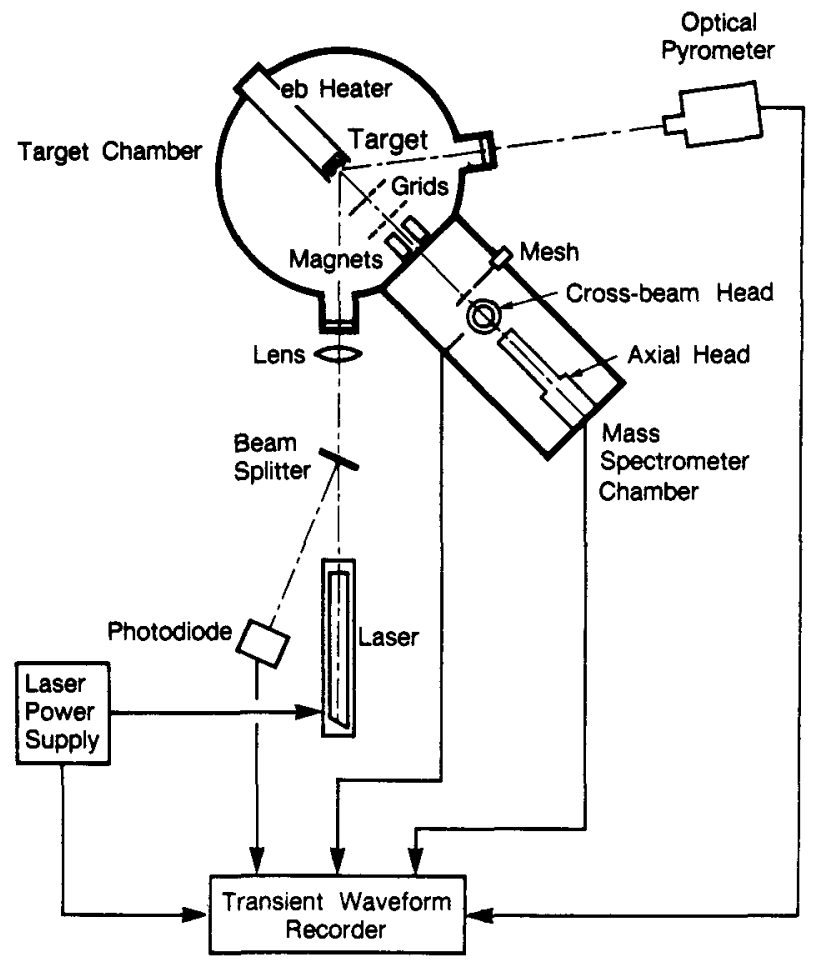

Fig. 3. Sketch of laserpulsing apparatus with pyrometric temperature measurement and mass spectrometric detection.

- Pulse power densities must be low enough to avoid significant emission of radiation from the hot plume at the wavelength of the optical pyrometer. Also, the plume should be optically transparent to the thermal radiation originating from the surface[35]. This requirement effectively limits the maximum temperatures which can be accurately determined by optical pyrometer to somewhat over $4000^{\circ} \mathrm{C}$ for refractory targets which have relatively low vapor pressures[26]. The limiting temperature would be considerably lower for aluminum, for example.

- The radial power density distribution in the laser beam should be flat enough that the pyrometer view spot intercepts a circle which is uniformly heated. Pyrometer view spots are on the order of a millimeter, so that the temperature of targets irradiated by highly-focussed laser beams cannot be measured. Conventional laser beams(e.g., from a Nd-glass laser operated in the conventional mode) produce a pulse which is uniform over about 7 - $9 \mathrm{~mm}$.

One method of assessing the accuracy of the surface temperature measurement is to compute the transient from a first-principles, no-adjustable-parameter heat transfer calculation. Such a calculation is tractable if the experiment satisfies a few simple restraints.

First, the temperature should depend only on time and depth beneath the surface but not on radial position in the solid. The depth of the laser-heated zone is of the order of $\left(\mathrm{Kt}_{\mathrm{p}}\right)^{1 / 2}$ where $\mathrm{K}$ is the thermal diffusivity of the condensed phase and $t_{p}$ is the width of the pulse. For millisecond-scale pulses in insulators, the thermal penetration depth is of the order of a few tens of microns. If the radius of the uniformly-irradiated spot is much larger than this depth, radial heat conduction can be neglected in the analysis. In addition, a large, uniformly-illuminated spot tends to suppress radial movement of the liquid[23,35]. This permits the radial convection term in the energy equation to be neglected and justifies the assumption of a flat surface.

Second, if the thermal penetration depth is significantly larger than the optical absorthion length(as it usually is for long pulses), the laser can be considered to be a surface rather than a volume heat source.

Third, the reflectivity $(R)$ of the surface at the wavelength of the laser light must be known in order to convert the specifiable incident laser power density on the surface, $q_{\text {inc }}$, to the absorbed power density,

$$
\mathrm{q}_{\mathrm{abs}}=(1-\mathrm{R}) \mathrm{q}_{\mathrm{inc}}
$$


which drives the heat transport process. The reflectivity of metals and ceramics decrease with increasing temperature[37,38].

Fourth, the temporal shape of the laser pulse must be known. The average power density trace is measured by the photodiode(Fig. 3). However, conventional-mode lasers actually produce a series of very narrow triangular pulses about one half microsecond wide and $1.5 \mu$ s apart. This heat input results in a surface temperature which oscillates about the value that a smooth pulse of the same power density as the average of the spikes would produce[39]. The amplitude of the oscillations depends on the physical properties chosen for the calculation, but appears to be from 50 to $100 \mathrm{~K}$ for most materials; in effect the surface has a considerable amount of thermal inertia, and unless a special effort is made, neither the pyrometer nor the mass spectrometer will respond to these oscillations.

When strong vaporization and high temperatures are involved, the nonlinearities inherent in the heating process preclude application of standard analytical solutions of the heat conduction equation. Only in the case of surface melting or annealing are such solutions sufficiently accurate. Nonlinear behavior of the governing equations appears in temperature-dependent thermal properties and in the necessity of accounting for the heat effect of vaporization at the surface and melting at the liquid-solid interface.

With the above restrictions, the energy equation in the condensed phase is given by[6]:

$\rho \bar{C}_{p}\left(\frac{\partial T}{\partial t}-u \frac{\partial T}{\partial z}\right)=\frac{\partial}{\partial z}\left(k \frac{\partial T}{\partial z}\right)$

where $\rho$ is the density of the solid or liquid at the surface, $\mathrm{k}$ is the thermal conductivity and $\bar{C}_{p}$ is an effective heat capacity. The latter provides a reasonably accurate method for accomodating the heat effect of fusion without requiring the more detailed treatment of conduction in a moving two-phase system[40]. The function:

$\bar{C}_{p}=C_{p}+\frac{\Delta H_{F}}{\sqrt{\pi} \Delta T_{F}} \exp \left(-\left(\frac{T-T_{F}}{\Delta T_{F}}\right)^{2}\right)$

spreads the melting discontinuity over a small but nonzero temperature range about the melting point $T_{F}$ while correctly accounting for the enthalpy of fusion, $\Delta \mathrm{H}_{\mathrm{F}}$. The true heat capacity of the condensed phase is $\mathrm{C}_{\mathrm{p}}$. The temperature range over which the heat of fusion is spread, $\Delta T_{F}$, is chosen so that the partially melted zone occupies a small spatial region compared with the depth of penetration of the thermal disturbance, but large enough to avoid instabilities in the numerical solution of $\mathrm{Eq}(6)$. This method is acceptable for the present application because the fraction of the laser input energy required to melt the upper layer of the solid is small. The accuracy of this approximte method of treating melting has been tested by comparing the results with standard solutions to problems of conduction with phase change[41].

The second term in the parentheses on the left hand side of $\mathrm{Eq}(6)$ represents recession of the surface due to evaporation given by $\mathrm{Eq}(3)$ (usually only one evaporating species is considered). The backscattering factor in a gasdynamic blowoff can be included in this equation.

The surface boundary condition for $\mathrm{Eq}(6)$ is given by the energy balance:

$q_{a b s}=-k\left(\frac{\partial T}{\partial z}\right)_{0}+\rho u \Delta H_{V}+\varepsilon \sigma\left(T_{s}^{4}-T_{a m b}^{4}\right)$

where $\Delta H_{V}$ is the heat of vaporization, $\varepsilon$ is the emissivity of the surface, $\sigma$ is the Stephan-Boltzmann constant and $\mathrm{T}_{\mathrm{amb}}$ is the temperature of the surroundings of the $\operatorname{system}(300 \mathrm{~K})$. In these equations the surface temperature is denoted by $\mathrm{T}_{\mathbf{s}}$. The kinetic energy carried off by the departing vapor molecules has been neglected in $\mathrm{Eq}(8)$. However, their momentum is the source of the recoil pressure discussed earlier.

The remaining conditions for $\mathrm{Eq}(6)$ are the initial condition

$T(z, 0)=T_{0}$

where $T_{0}$ is the initial temperature of the solid. The condition representing a semi-infinite medium is:

$T(\infty, t)=T_{0}$ 
The above set of equations can be solved numerically by standard partial differential equation solvers in commercial software libraries. The principal output is the time variation of the surface temperature, although subsurface temperatures and the location of the melting front can be extracted if desired.

Figure 4 shows the measured and calculated temperature histories following a laser pulse with a peak power density of $10^{4} \mathrm{~W} / \mathrm{cm}^{2}$ on uranium dioxide at an initial temperature of $1600 \mathrm{~K}$. The comparison is based on the temperature rise normalized to the temperature rise at the peak temperature in order to illustrate two aspects of the results. First, the agreement between the measured and predicted times of the maximum surface temperature is good. The temperature reaches a maximum at $354 \mu$ s following onset of laser irradiation whereas the laser power density peaks at $320 \mu \mathrm{s}$. Second, the shape of the measured transient is in good accord with the predicted shape. The principal uncertainty in comparing the experimental and theoretical absolute values of the maximum surface temperature $T_{\text {smax }}$ is in assignment of the surface reflectivity. Fortunately this quantity has been measured for $\mathrm{UO}_{2}$ [38]. For pulses which produced measured maximum surface temperatures from 2380 to $3670 \mathrm{~K}$, the calculated values average about $130 \mathrm{~K}$ lower than the observed values[35].

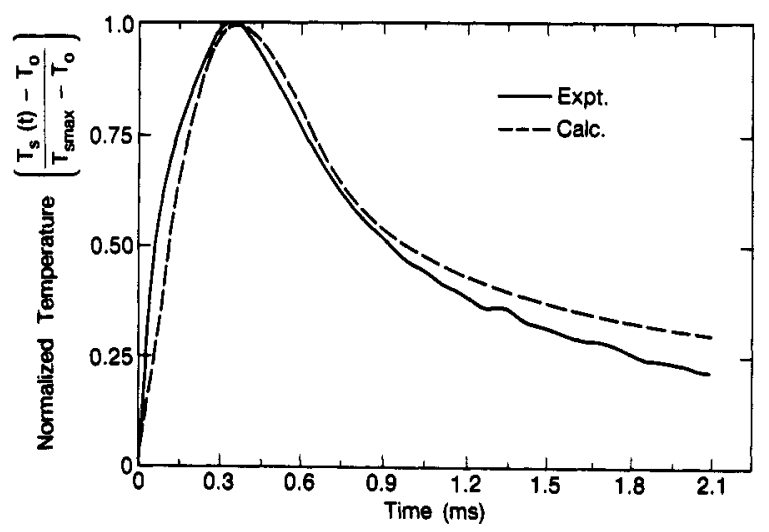

Fig. 4. Measure and calculated normalized surface temperature rises for a laser pulse with a peak power density of $10^{4} \mathrm{~W} / \mathrm{cm}^{2}$ incident on a $\mathrm{UO}_{2}$ surface of initial temperature $1600 \mathrm{~K}$. The peak surface temperature measured in this experiment was $2400 \mathrm{~K}$.

The generally good agreement between the observed and computed temperature transients for laser pulsing of uranium dioxide suggests that measuring surface temperatures by optical pyrometry is reasonably accurate and can be generally applied for millisecond-scale laser pulses on most materials, at least those for which the high-temperature reflectivity is either known or so close to unity that errors in this parameter do not significantly affect the measurement. The limit imposed by the onset of plasma emission or shielding of the surface must also be respected.

\section{Species detection by mass spectrometry}

One of the principal applications of mass spectrometric detection of the vapor emitted from a laser-heated target is measurement of the thermochemical properties of refractory substances at temperatures beyond the reach of conventional methods. Both the total vapor pressure and the various molecular species in the vapor are of particular interest. In addition, mass spectrometers can be used as time-of-flight detectors to determine the energies of the species emitted from the surface.

Use of a mass spectrometer for this purpose requires extensive calibrations of instrumental sensitivity to the molecules detected and estimation of their fragmentation patterns. These problems are the same as those that have to be dealt with in conventional Knudsen-cell mass spectrometric vaporization studies.

A convenient method of comparing theory and experiment is by calculation and measurement of the number density of particular species in the ionizer. Since mass spectrometers are density-sensitive devices, the instantaneous output signal $V(t)$ is proportional to the instantaneous number density in the ionizer, $n(t)$, or:

$(n(t))_{\exp }=K V(t)$

where $\mathrm{K}$ is an instrumental constant that depends on the geometries of the flight path between the target and the mass spectrometer, the ionizing electron current, the fragmentation pattern, various mass spectrometer efficiency 
factors and the gain of the electron multiplier. Calibration can be performed by steady-state vaporization of the material or by using laser pulses of sufficiently low energy density that the maximum temperature of the surface is in the range in which the vapor pressure is known.

As explained earlier, the number density in the vapor downstream of the target is not greatly dependent on the extent of intermolecular collisions in the blowoff(provided that only thermally-driven evaporation occurs). For free-molecule vaporization, the number density in the vapor at a distance $L$ from the target is given by a convolution of the vapor pressure (driven by the surface temperature transient $T_{s}(t)$ ) and the Maxwellian speed distribution of the emitted molecules[42]:

$$
n(t)=\frac{A_{s} L}{k}\left(\frac{m}{2 \pi k}\right)^{3 / 2} \int_{0}^{t} \frac{P\left\{T_{S}(\tau)\right\}}{\left\{T_{S}(\tau)\right\}^{5 / 2}(t-\tau)^{4}} \exp \left(-\frac{m L^{2}}{2 k T_{S}(\tau)(t-\tau)^{2}}\right) d \tau
$$

Here $A_{s}$ is the area of the irradiated spot on the target, $P\left(T_{s}\right)$ is the vapor pressure of the material at the temperature of the surface (only one component is assumed) and $k$ is the Boltzmann constant. Equation(12) is a theoretical time-of-arrival curve, which can be computed if the vapor pressure is known. It is strictly speaking valid only for free-molecule flow, and indeed it does not correctly predict the arrival time in a gasdynamic blowoff. However, if only the quantity of material removed is of interest, the maximum value of $n$ or its integral over the entire pulse are nearly equal for free-molecule and gasdynamic evaporation regimes. In the latter case, however, the right hand side of $\mathrm{Eq}(12)$ must be reduced by the backscattering factor discussed earlier.

\section{RESULTS - URANIUM DIOXIDE}

Figure 5 shows an experimental trace of the $U \mathrm{O}_{2}^{+}$signal for a relatively weak laser pulse on solid $\mathrm{UO}_{2}$ (wavy line) and the predicted time-of-arrival curve from $\mathrm{Eq}(11)$ (smooth curve). The maximum surface temperature attained in this pulse was $2400 \mathrm{~K}$, where the vapor pressure is too low to initiate a gasdynamic blowoff. The theoretical and experimental curves in Fig. 5 have been normalized at their peak values(the normalization factor is the calibration constant for this species). The excellent fit of the two curves, particularly the time at which the maximum signal occurs and the overall shape, demonstrates that the evaporation mechanism is classical free-molecule flow with the emission rate given by the Hertz-Langmuir equation.

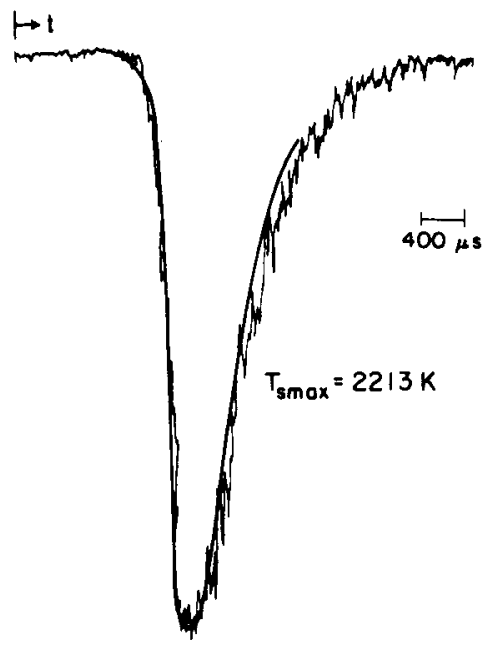

Fig. 5. Mass spectrometer signals (wavy trace) for $10_{2}^{+}$following a laser pulse which resulted in a maximum surface temperature of $2213 \mathrm{~K}$. The smooth curve is calculated from Eq(72). The laser was fired at the time represented by the vertical line in the upper left hand corner of the diagram.

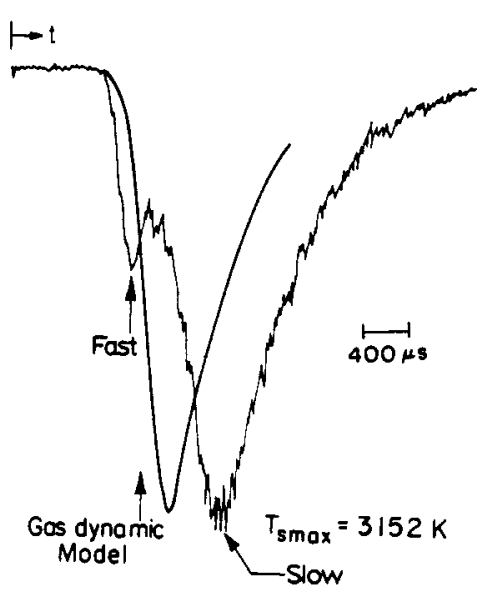

Fig. 6. Experimental signals and freemolecule flow prediction similar to those shown in Fig. 5 but for a pulse giving a maximum surface temperature of $3152 \mathrm{~K}$.

Figure 6 shows a similar comparison for a laser shot which produced a peak surface temperature just below the 
melting point of $\mathrm{UO}_{2}$. The maximum temperature for this pulse is greater than the critical temperature at which the flow regime is calculated to switch from free-molecular to gasdynamic(which is about $2600 \mathrm{~K}$ ). The experimental trace shows distinctly nonMaxwellian behavior. Two peaks are observed. One(labeled "fast" in Fig. 6) arrives earlier than expected for free-molecule vaporization and may be due to gasdynamic acceleration of the flow. The larger peak is slower than expected from free molecule vaporization. Neither peak contains any ions, as was verified by turning off the electron current in the ionizer and also by placing magnetic and electric deflection plates around the vapor plume between between the target and the mass spectrometer.

In addition to the clear departure of the shape of the time-of-arrival curve from that expected for equilibrium collisionless vaporization, the quantity of vapor emitted, as measured by the maximum signal, is a factor of nearly 100 smaller than predicted by $\mathrm{Eq}(12)$. This phenomenon is shown in Fig. 7, wherein the maximum signal is plotted as a function of various measures of the strength of the laser pulse. Up to a peak surface temperature of about $2400 \mathrm{~K}$, the measurement conforms to equilibrium vacuum vaporization. The slope of a line through the data points in this regime is unity and the level of the signal gives the calibration factor. At higher temperatures, however, the signal levels off and practically ceases to increase with laser pulse energy. It should have continued to increase along the extrapolated line of the low-temperature results.

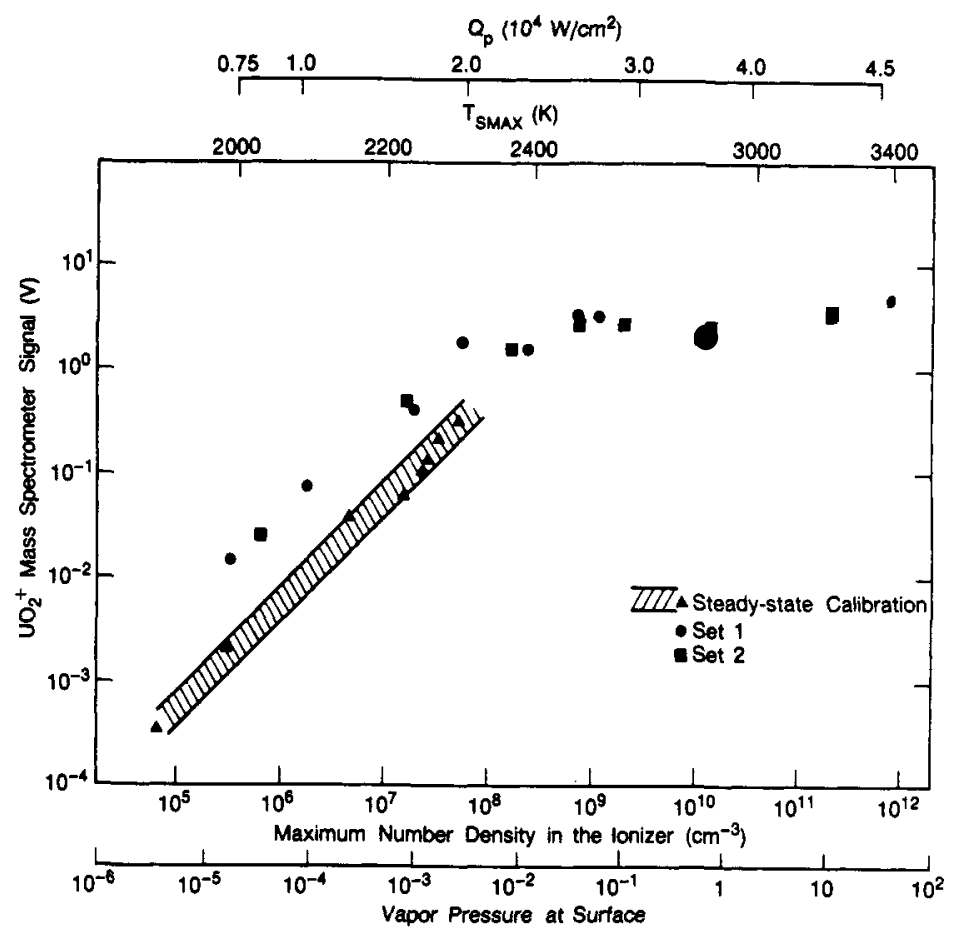

Fig. 7. Maximum $\mathrm{NO}_{2}^{+}$signals from the mass spectrometer for laser pulses of varying strength. $2 p$ is the peak absorbed power density, and $T_{\text {Smax }}$ is the measured maximum surface temperature in the pulse. The scale designating the maximum number density in the ionizer of the mass spectrometer was calculated from $E q(12)$ and the vapor pressure is that of $\mathrm{UO}_{2}$ at the peak surface temperature. The hatched area represents the range of results of the steady-state calibrations.

A number of possible experimental artifacts were examined and discarded as explanations of this discrepancy. This behavior is also observed with $\mathrm{ZrO}_{2}$ but not for chromium[29]. The loss of signal at high surface temperatures does not mean that the quantity vaporized is smaller than expected theoretically; it simply may mean that the vapor is not principally $\mathrm{UO}_{2}$. In fact, neutron activation analysis of the deposit on collector plates near the target was consistent with vaporization according to $\mathrm{Eq}(1)$.

The best explanation of the phenomon seen in Fig. 7 is that $\mathrm{UO}_{2}$ nucleates and grows clusters which, by virtue of their larger mass, are not detected as $\mathrm{UO}_{2}^{+}$. This explanation cannot be tested for $\mathrm{UO}_{2}$ because even the dimer $\left(\mathrm{UO}_{2}\right)_{2}$ is beyond the mass range of the instrument. However, laser pulsing of $\mathrm{MgO}$ at temperatures above $1700 \mathrm{~K}$ showed the presence of masses corresponding to $(\mathrm{MgO})_{\mathrm{n}}$, with $\mathrm{n}=2,3,6$, and 7. The "slow" peak in Fig. 6 is believed to be due to fragmentation of slowly-moving $\left(\mathrm{UO}_{2}\right)_{2}$ clusters to produce small quantities of $\mathrm{UO}_{2}^{+}$.

A possible reason that metals such as chromium do not show the anamolous levelling-off seen in Fig. 6 whereas oxides such as $\mathrm{UO}_{2}$ and $\mathrm{ZrO}_{2}$ do is the difficulty of nucleating clusters from atomic monomers. Small clusters formed from single-particle monomers have fewer degrees of vibrational and rotational freedom to absorb the 
binding energy of cluster formation compared to clusters formed from molecular monomer units. Gaponov et. al.[30] offer a different explanation. The reflectivity of metals for the $1.06 \mu \mathrm{m}$ radiation from a $\mathrm{Nd}$ laser can be as large as $70-90 \%$, whereas ceramic oxides have much lower reflectivies(for $\mathrm{UO}_{2}$ the value is approximately $15 \%$ ). The laser light reflected by the metal surface can be absorbed by clusters and cause them to revaporize. Even for low-reflectivity targets, a similar effect can be obtained by reducing the angle between the laser axis and the surface normal, which will cause the laser light to intercept more of the expanding plume than if it impinged on the target at an oblique angle. Gaponov et. al.[30] proposed a criterion for condensation in the expanding gasdynamic plume to occur; The product of the vapor density corresponding to the vapor pressure at the surface temperature and the radius of the irradiated spot should be greater than $10^{17}$ to $10^{18} \mathrm{~cm}^{-2}$. When this criterion was met for normal-mode laser evaporation of bismuth, submicron-size spherical particles were observed on collector plates a few centimeters downstream of the target. A detailed discussion of condensation in laser-driven gasdynamic flows is given by Anisimov et al[43].

Condensation in a gasdynamic plume is particularly bothersome because it occurs when the surface is at high temperatures where the thermochemical information is needed. There are several options for dealing with this problem. First, nothing may need to be done if only ratios of vapor species concentrations is desired rather than absolute values of the equilibrium partial pressures(or vaporization rates). There is evidence from laser-pulsing of $\mathrm{UO}_{2}$ and $\mathrm{UC}[14,29]$ that the ratios of the vapor species do not change drastically as the blowoff changes from free-molecule to gasdynamic in nature. The high temperature equilibium thermochemistry at the surface conditions may be preserved in spite of clustering and cooling during the expansion. Figure 8 compares the predicted and measured ratios $\mathrm{UC}_{2} / \mathrm{U}$ and $\mathrm{C}_{3} / \mathrm{C}$ as functions of the maximum surface temperature reached in a series of shots of different laser energy density. The ratios of the uranium-bearing species agree with the thermochemical models at low temperature , but not at high temperature. The opposite behavior is exhibited by the carbon species. No $C_{2}$ was detected, although this species was predicted thermochemically to be present at partial pressures comparable to those of $C_{3}$. The work of Hastie and Bonnell, on the other hand, suggests that the relative amounts of the $C_{n}$ species emitted from laser-pulsed graphite is in accord with equilibrium expectations[32].

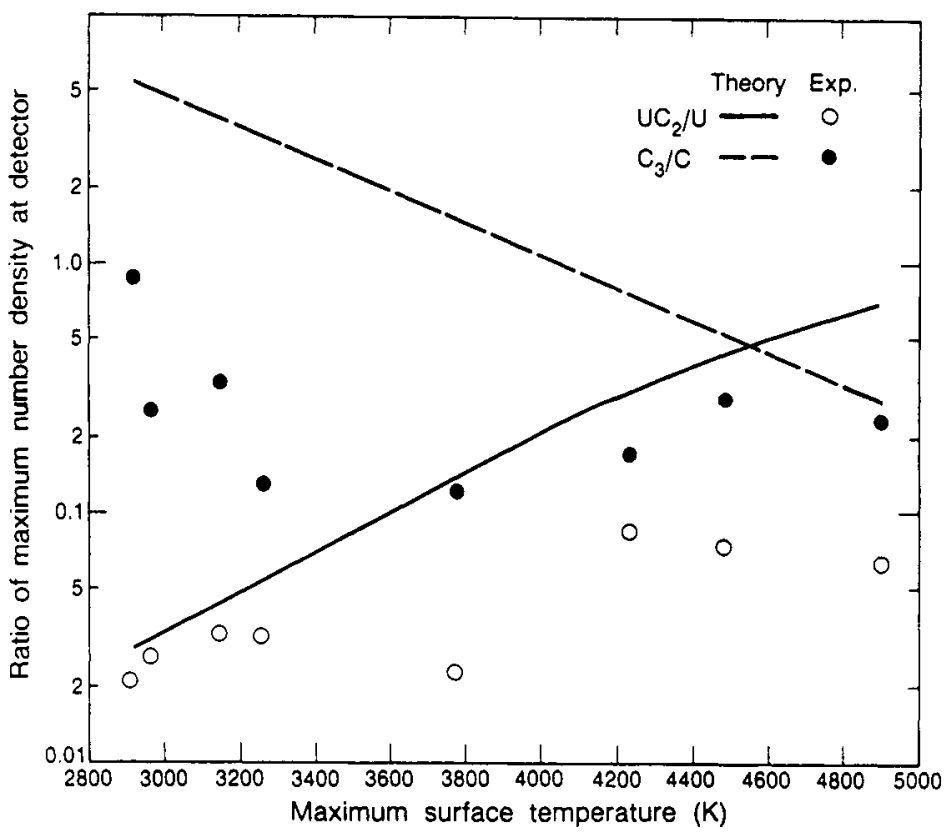

Fig. 8. Measured and theoretical ratios of uranium species and carbon species due to laser-pulsing of uranium carbide. The maximum surface temperature is measured.

Second, it may be possible by experimental means to prevent clustering during the expansion. Two such methods have been suggested by Gaponov et. al.[30], namely using small irradiated spots and tilting the incident laser beam as close to the normal as possible. It may also be possible to evaporate clusters using a second laser crossing the expanding plume, as has been demonstrated for $\mathrm{SF}_{6}$ clusters a supersonic jet[44]. Even more simply, illuminating the vapor plume by a laser flash lamp coiled around the vapor plume may deliver enough energy to dissipate the condensate.

In any case it is clear that more work is needed to elucidate the nature of formation of clusters by condensation of vapor in the gasdynamic expansion of a plume formed by laser irradiation of solid targets. The distribution of 
cluster sizes in the plume is information of prime importance. This should be accessible by using the reflectron time-of-flight mass spectrometer[45].

\section{CONCLUSIONS}

Laser irradiation with millisecond-width pulses is a useful technique for studying the thermochemistry of refractory solids at very high temperatures. For power densities less than about $10^{6} \mathrm{~W} / \mathrm{cm}^{2}$, the vaporization rate is closely represented by the Hertz-Langmuir formula. Within this regime, two types of vapor blowoff have been identified, namely free-molecule and gasdynamic expansions. The latter regime may be accompanied by nucleation and growth of weakly-bound clusters but the relative concentrations of the various molecular species in the vapor appears to be characteristic of conditions at the emitting surface(i.e., composition and temperature).

In contrast to short(nanosecond-scale) laser pulses, the surface temperature transients created by long laser pulses can be measured with reasonable accuracy by optical pyrometry, which is essential for thermochemical investigations.

The main unresolved questions connected with thermally-driven vaporization by long laser pulses are the extent of ionization of the vapor and the conditions under which cluster formation occurs. Recently-developed mass spectrometers and novel experimental techniques should lead to a better understanding of clustering and to development of methods of avoiding this phenomenon.

Pulsed-laser vaporization is also a promising method for producing thin films of refractory materials for use in integrated circuits and high- $T_{c}$ ceramic superconductors. Recent work in this field has concentrated on short, high-power-density laser pulsing, but the use of long laser pulse processing may have some advantages. Chief among these are avoidance of chunk or droplet ejection from the irradiated spot and elimination of high-energy ion emission, both of which can lead to less-than-optimum characteristics of the deposited films.

\section{Acknowledgement}

This work was supported in part by the Director of the Office of Energy Research of the Office of Basic Energy Science, Material Science Division of the U. S. Department of Energy under contract number DE-AC03-76sf00098.

\section{REFERENCES}

1. Proceedings of conference on "Applications of Lasers in Materials Processing" , E. A. Metzbower, Ed., Washington, D. C., American Society for Metals (1979)

2. M. von Allmen, "Laser Beam Interactions with Materials", Springer-Verlag (1986)

3. L. Woste, in "Contribution of Cluster Physics to Materials Science and Technology", J.Davenas and P. M. Rabette, Eds, pp 1 -50 (1982)

4. A. Inam, X. D. Wu, T. Venkatesan, S. B. Ogale, C. C. Chang and D. Dijkkamp, Appl. Phys. Lett., 51, 1112 (1987)

5. G. M. Rosenblatt, Accounts of Chem. Research, 9, 169 (1976)

6. C. H. Tsai and D. R. Olander, J. Nucl. Mater. 137, 279 (1986)

7. W. Breitung, Kernforschungszentrum Karlsruhe Report No. KfK-2240 (1976)

8. M. Murakami and K. K. Oshima, in Rarefied Gas Dynamics, M. Becker and M. Fiebig, Eds, Paper No. F.6, (DFVLR, Porz-Wahn, Germany, 1974)

9. C. H. Tsai and D. R. Olander, Phys. Fluids 30, 386 (1987)

10. J. B. Anderson, in "Molecular Beams and Low-Density Gasdynamics", P. P. Wegener, ed., Chap 1, Marcel Dekker, New York (1974) 
11. S. I. Anisimov, Sov. Phys. JETP 27, 182 (1968)

12. C. Cercignani, in Rarefied Gas Dynamics, S. Fisher, Ed, p 305, AIAA, New York (1981)

13. R. Kelly and R. W. Dreyfus, Surf. Sci. 198, 263 (1988)

14. F. Tehranian and D. R. Olander, High Temp. Sci. in press

15. J. F. Ready, J. Appl. Phys. 36, 462 (1965)

16. F. P. Gagliano and U. C. Paek, Appl. Opt. 13, 274 (1974)

17. V. I. Mazhukin and A. A. Samokhin, Sov. J. Quant. Electron. 14, 1608 (1984)

18. F. W. Dabby and U. C. Paek, IEEE J. of Quant. Electron., Vol. QE-8, 106 (1972)

19. B. M. Kozlov, B. B. Krynetskii and A. A. Samokhin, Sov. J. Quant. Electron., 4, 1303 (1975)

20. R. Kelly, J. J. Cuomo, P. A. Leary, J. E. Rothenberg, B. E. Braren and C. F. Aliotta, Nucl. Instr. and Meth. B9, 329 (1985)

21. J. E. Rothenberg and R. Kelly, Nucl. Instru. and Meth. B1, 291 (1984)

22. R. Kelly and J. E. Rothenberg, Nucl. Instr. and Meth. B7/8, 755 (1985)

23. J. Magill, C. Ronchi, J-F Babelot, K. A. Long and R. W. Ohse, High Temp. - High Press., 12, 503 (1980)

24. R. W. Ohse, J-F. Mabelot, C. Cercignani, J-P Hiernaut, M. Hoch, G. J. Hyland and J. Magill, J. Nucl. Mater. 130, $165(1985)$

25. J. F. Ready, "Industrial Applications of Lasers" , chap. 13, Academic Press (1978)

26. M. Bober and H. U. Karow, 7th Symp. on Thermophysical Properties, NBS, Washington, D. C. (1977)

27. J. A. McKay and J. T. Schriempf, Ref. 1, p 55

28. R. W. Dreyfus, R. Kelly and R. E. Walkup, Nucl. Instr. and Meth. B23, 557 (1987)

29. D. R. Olander, S. K. Yagnik and C. H. Tsai, J. Appl. Phys., 64, 2680 (1988)

30. S. V. Gaponov, A. A. Gudkov and A. A. Fraerman, Sov. Phys. Tech. Phys., 27, 1130 (1983)

31. R. A. Olstad and D. R. Olander, J. Appl. Phys, 46, 1509 (1975)

32. J. W. Hastie, D. W. Bonnell and P. K. Schenk, High Temp. - High Press., 20, 73 (1988)

33. M. Bober, H. U. Karow and K. Schretzmann, Nucl. Technol. 26, 237 (1975)

34. R. W. Ohse, J-F. Babelot, A.Frezzotti, K. A. Long and J. Magill, High Temp - High Press. 12, 537 (1980)

35. S. K. Yagnik and D. R. Olander, J. Nucl. Mater. 154, 253 (1988)

36. B. M. Zhiryakov, A. I. Korotchenko, N. I. Popov and A. A. Samokhin, Sov. J. Quant. Electron. 13, 763 (1983)

37. K. Park and W. T. Walter, Ref. 1 p. 21

38. H. U. Karow and M. Bober, Proceedings of Symp. on the Thermodynamics of Nuclear Materials, Vol. 1, p. 155, Julich, IAEA (1979) 
39. R. A. Olstad, "Mass Spectrometric Investigation of Laser-Induced Vaporization of Binary Solid Compounds", PhD Thesis, LBL-1177 (1972)

40. A. M. Hassanein, G. L. Kulcinski and W. G. Wolfer, J. Nucl. Mater., 103\&104, 321 (1981)

41. D. R. Olander and Y. S. Kim, in preparation

42. R. A. Olstad and D. R. Olander, J. Appl. Phys. 46, 1499 (1975)

43. S. I. Anisimov, Y. A. Imas, G. S. Romanov, and Y. U. Khodyko, "Effect of High-Power Radiation on Metals", National Tech. Info. Service, Springfield, VA (1970)

44. R. Rechsteiner, R. Monot, L. Woste, J. M. Zellweger and H. van den Bergh, Helv. Phys. Acta, 54, 282 (1981)

45. B. A. Mamyrin, V. I Karataev, D. V. Shmikk and V. A. Zagulin, Sov. Phys. JETP, 37, 45 (1973) 